\title{
A pedicled bone graft from the acromion: an anatomical investigation regarding surgical feasibility
}

\author{
Beat Kaspar Moor, MD ${ }^{a, *}$, Georges Kohut, MD ${ }^{a}$, Samy Bouaicha, MD ${ }^{a}$, \\ Silke Grabherr, $\mathbf{M D}^{\mathbf{b}}$, Emanuel Gautier, $\mathbf{M D}^{\mathrm{a}}$, Mathias Bergmann, $\mathbf{P D}^{\mathrm{c}}$, \\ Nicholas Marcer, $M^{c}$, Valentin Djonov, MD $^{c}$
}

\author{
${ }^{a}$ Department of Orthopaedic Surgery, Hôpital cantonal de Fribourg, Fribourg, Switzerland \\ ${ }^{b}$ University Center of Legal Medicine Lausanne-Geneva, University of Lausanne, Lausanne, Switzerland \\ ${ }^{c}$ Institute of Anatomy, Department of Medicine, University of Fribourg, Fribourg, Switzerland
}

\begin{abstract}
Objective: To investigate the technical feasibility of harvesting a vascularized bone graft from the acromion pedicled on the acromial branch.

Background: Complex fractures of the proximal humerus may result in partial or total avascular necrosis of the head fragment. Treatment of avascular necrosis of the humeral head is dependent upon the stage of disease as well as the dimension and location of necrosis. In general, the outcome is poor and complete restoration of the shoulder function is rarely attained. Contrary to osteonecrosis of carpal bones (where vascularized bone grafts have been routinely carried out for decades), reports of analogous procedures at the humeral head are anecdotal.

Methods: Based on selective post-mortem computer-tomographic angiography of 5 and the dissection of 30 embalmed human cadaver shoulders, we describe the anatomy of the acromial branch of the thoracoacromial trunk. The main focus was the constancy of its anatomical course, its dimensions and potential use as a nutrient vessel for a pedicled bone graft from the acromion.

Results: The course of the acromial branch revealed a constant topographic relationship to anatomical landmarks. Its terminal branches reliably supplied the anterior part of the acromion. The vascularized bone graft could be sufficiently mobilized to allow tension-free transfer to the humeral head as well as to the lateral two-thirds of the clavicle.

Conclusion: We demonstrated the feasibility of vascularized bone graft harvesting from the acromion. This technique could be a joint-preserving procedure for osteonecrosis of the humeral head or may assist in the revision of a clavicular pseudoarthrosis.

Level of evidence: Anatomic Study, Cadaver Dissection.
\end{abstract}

Keywords: Acromion; vascularized bone graft; revascularization; acromial branch; thoracoacromial trunk; avascular necrosis

\footnotetext{
*Reprint requests: Beat Kaspar Moor, MD, Department of Orthopaedic Surgery, Hôpital cantonal de Fribourg, 1708 Fribourg, Switzerland.

E-mail address: bmoor@gmx.ch (B.K. Moor).
}

The use of vascularized bone grafts is increasing worldwide. Ever since 1975 , when Taylor et al ${ }^{32}$ carried out the first free transfer of a vascularized fibula, vascularized bone grafts have been suggested for the reconstruction of massive 
segmental bone defects mainly after trauma, chronic osteomyelitis, and resection of malignant bone tumors, as well as for the treatment of atrophic nonunion or the revascularization of avascular osteonecrosis. $1,18,21,31,37,38,40$

Many vascularized bone-transfer sites are available around the hand and wrist, but descriptions of pedicled bone transfers at the shoulder are only anecdotal. However, there are several situations in which vascularized bone grafts at this location could be beneficial. These include revision of an atrophic nonunion of the clavicle (reported incidence of $0.1-1.9 \%^{13,23,29}$ ) and the revascularization of an ischemic head fragment after complex fracture of the proximal humerus (reported incidence of $15-30 \%{ }^{17}$ ).

Vascular damage with active bleeding may seriously compromise visualization of the operative area during shoulder arthroscopy. In particular, during trimming of the anterolateral acromion, bleeding from ramifications of the acromial branch of the thoracoacromial trunk is frequently encountered. ${ }^{41}$ This acromial branch with its "coracoacromial arterioles" is a potential source of bleeding during arthroscopic acromioplasty; so, we hypothesized that it could be used as nutrient artery for a vascularized bone graft from the acromion.

The purpose of the present anatomical study was to investigate the technical feasibility of harvesting a vascularized bone graft from the acromion, pedicled on the acromial branch. The primary focus lies on the constancy of a distinct acromial branch, as well as its dimensions and anatomical course. We also evaluated its potential use as a nutrient vessel for a pedicled bone transfer from the acromion.

\section{Materials and methods}

All investigations were carried out on 35 Thiel-fixed $^{33}$ shoulders of the human cadavers of 9 females and 10 males, according to medical-ethical guidelines and recommendations for working with cadaver material. ${ }^{5}$ The mean age at the time of death was 76 years (range, 52-93). To preserve the anonymity of the donors, information such as personal identity and medical history was not disclosed. Shoulders were removed from the trunk according to a forequarter amputation. Only specimens without evidence of previous surgery or a visible abnormality were included. Before dissection, all shoulders were allocated to 1 of 3 groups, as described below.

\section{Group I (post-mortem CT angiography)}

Five intact shoulders were used to investigate the anatomical course of the acromial branch in situ in nondissected specimens with preserved topographic anatomical relationships. The acromial branch was identified via the thoracic cavity near its origin from the thoracoacromial trunk and selectively cannulated with a $1.8 \times 45 \mathrm{~mm}$ intravenous catheter (BD Venflon ${ }^{\mathrm{TM}}$ Pro $16 \mathrm{G}$; $\mathrm{BD}$, Haryana, India). Under manual pressure, $10 \mathrm{~mL}$ of a radioopaque solution (Angiofil ${ }^{\circledR}$; Fumedica AG, Muri, Switzerland) was injected. ${ }^{9}$ In all 5 specimens, post-mortem CT angiography on a CT Light Speed 8 unit (GE Healthcare; Milwaukee, WI,
USA) with the following parameters was carried out: collimation, $1.25 \mathrm{~mm}$; slice width, $1.25 \mathrm{~mm}$; reconstruction increment, $1.0 \mathrm{~mm}$. Review of primary 2-dimensional (2-D) images and 3-dimensional (3-D) reconstructions were done on a Advantage Workstation 4.3 (GE Healthcare, Milwaukee, WI, USA).

\section{Group II (vascular casting)}

In 12 specimens, arterial casting was carried out with a blue-dyed synthetic resin (polyurethane; Vasqutech Company, Zurich, Switzerland). The acromial branch was identified and selectively cannulated, as described for group I. Under manual pressure, $\sim 10 \mathrm{~mL}$ polyurethane was injected until perfusate flow was observed through the skin over the deltoid muscle. After polymerization of the polyurethane, the skin and subcutaneous tissues were removed. This was followed by release of the origin of the deltoid muscle and pectoralis major muscle. The acromial branch of the thoracoacromial trunk was identified at the medial edge of the pectoralis minor muscle and its course followed to the acromion. A volume of bone was harvested from the anterior acromion with a chisel; the bite was a little larger (but similar to) an acromioplasty as described by Rockwood and Lyons. ${ }^{27}$ To preserve the fragile coracoacromial arterioles, insertion of the coracoacromial ligament (CAL) had to be preserved; however, by doing so, the bone graft could be harvested under meticulous maintenance of its nutrient vessel. After graft mobilization, its cancellous bone was inspected for blue staining (indicating perfusion through ramifications of the acromial branch). Finally, the technical feasibility of a pedicled bone transfer to the humeral head and the clavicle was assessed.

\section{Group III (anatomical dissection)}

The constancy of the anatomical course of the acromial branch was evaluated in all stained (group II) specimens and in another 18 specimens without arterial casting. For that purpose, the topographic relationships of the acromial branch to anatomical landmarks that are readily detectable during surgical interventions were assessed. After dissection, as described for group II, the following distances were measured with a hand caliper graded in millimeters: (i) the interval from the acromial branch to the tip of the coracoid process in its longitudinal axis (coracoid ramus acromialis distance); (ii) the span between the vessel and the acromioclavicular joint (acromioclavicular joint ramus acromialis distance); (iii) the CAL was shown to be the main limiting factor for the range of graft transposition in group II, so its length was also determined (coracoacromial ligament length); and (iv) the diameter of the acromial branch was assessed at the level of the coracoid process.

Descriptive analyses were carried out with a commercial statistical package (Analyze It for Microsoft Excel). Testing for normality was performed with the Shapiro-Wilk test.

\section{Results}

\section{Group I (post-mortem CT angiography)}

In 1 specimen, the contrast solution leaked out, making further investigation impossible. In the remaining 4 shoulders, the course of the acromial branch could be visualized 


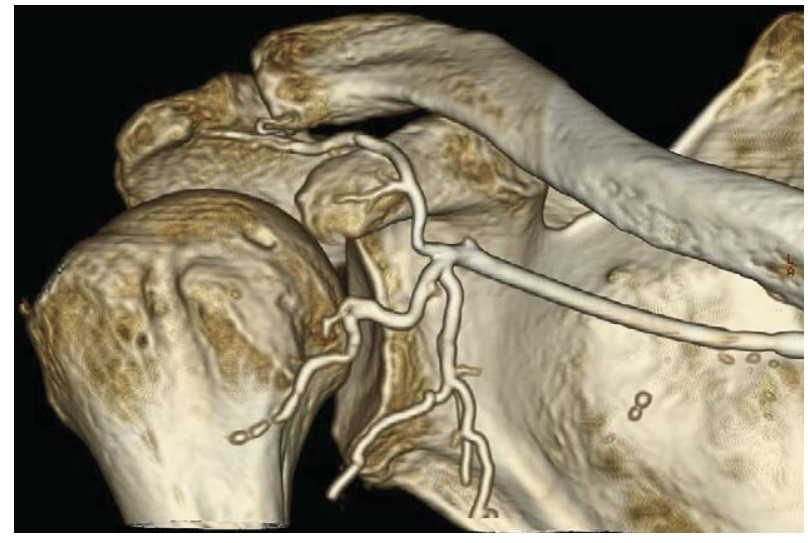

Figure 1 Three-dimensional reconstruction of post-mortem angiography reveals the topographic in-situ relationship of the acromial branch in a nondissected specimen of the right shoulder. Note the course of the artery over the coracoid process to the acromion. After passage over the coracoid process, it runs almost parallel to the lateral end of the clavicle.

(Fig. 1). After its origin from the thoracoacromial trunk, the acromial branch curved cranially and passed over the coracoid process. Further lateral, it ran almost parallel to the distal end of the clavicle. At the level of the acromioclavicular joint (ACJ), it divided into 2-3 branches. This technique did not permit visualization of the intraosseous course of the vessel in the acromion because of the limited resolution of the radiological examination. Furthermore, due to its similar radiopacity, it did not contrast sufficiently from the bone. The mean coracoid ramus acromialis distance was $21.4 \mathrm{~mm}$ (range, 19.8-23.2) and the mean acromioclavicular joint ramus acromialis distance was $8.5 \mathrm{~mm}$ (7.4-9.2). The mean vascular diameter of the acromial branch at the level of the coracoid process was $2.5 \mathrm{~mm}(2.0-2.7)$.

\section{Group II (vascular casting)}

The acromial branch was consistently identified at the medial border of the pectoralis minor muscle. After passage over the coracoid process, it ran parallel to the clavicle over the upper-third of the CAL (Fig. 2, A). At this level it was embedded into a compact layer of fatty tissue, accompanied by a dense venous rete. The whole package could be readily mobilized from the rear surface of the CAL and from the under-surface of the deltoid muscle. At the lateral end of the clavicle it gave origin to a small vessel, which crossed over the ACJ. After sending out a small branch to the lateral clavicle, it formed the acromial rete on the rear surface of the acromion. Finally, it anastomosed with the suprascapular artery. The remaining part of the acromial branch traveled further laterally before finally entering the deltoid muscle.

In 10 of 12 specimens, we found 2-3 terminal branches, which entered the acromion at its anterior border to supply its cancellous bone (Fig. 2, B). In the 2 remaining specimens in which these terminal branches could not be visualized, we noted extravasation of the polyurethane at the
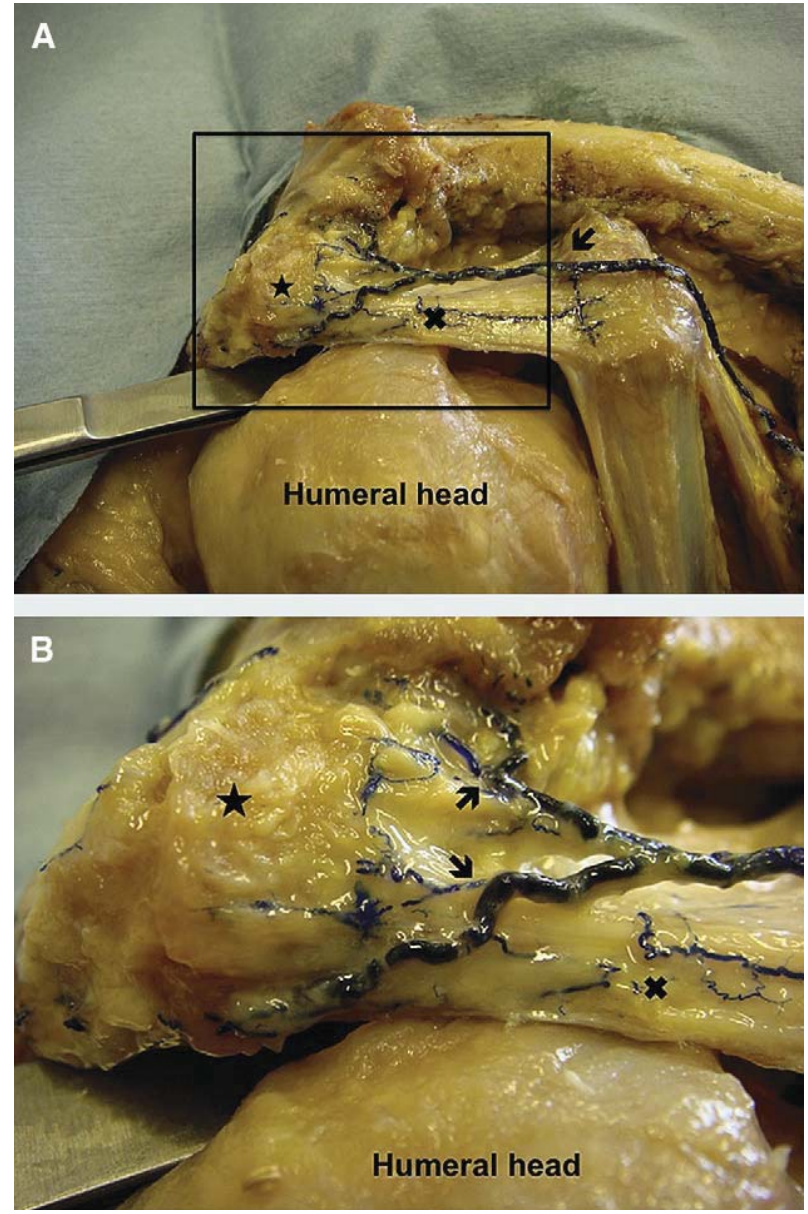

Figure 2 (A) Overview of a specimen of a right shoulder dissected in group II (vascular casting) demonstrating the course of the acromial branch over the tip of the coracoid process. (B) Closer view (rectangle in A) showing the acromial insertion of the coracoacromial ligament $(\times)$. There were consistently 2-3 coracoacromial arterioles (arrows), which entered the acromion $\left({ }^{*}\right)$ at its anterior border.

level of the ACJ. A too-high injection pressure probably caused rupture of these fragile terminal branches.

After ligation of the anastomosis to the acromial rete and the branch to the deltoid muscle, the bone graft could be readily harvested and mobilized. Its inspection revealed a dense vascular rete that infused the cancellous bone of the acromion (Fig. 3).

The CAL, which protected the acromial branch from traction forces during graft mobilization, was the main limiting factor of the transposition range. However, the distance was more than sufficient to allow tension-free transfer to the humeral head as well as to the lateral twothirds of the clavicle (Fig. 4).

\section{Group III (anatomical dissection)}

The course of the acromial branch was very constant, with a mean coracoid ramus acromialis distance of $23.2 \mathrm{~mm}$ 

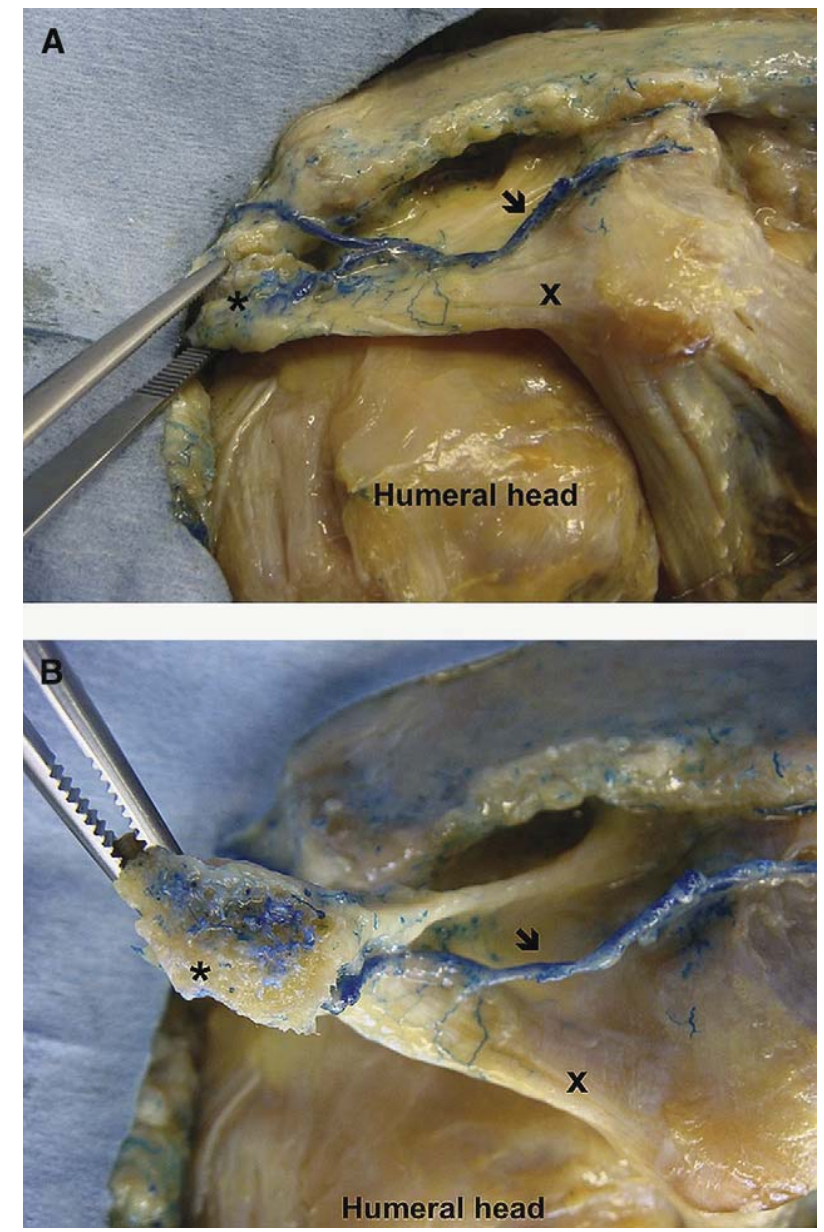

Figure 3 The anterolateral acromion of an injected right shoulder specimen is shown before (A) and after mobilization and rotation of the bone graft (B). (A) The bone cut was already carried out under maintenance of the acromial attachment of the coracoacromial ligament $(\times)$ and the acromial branch (arrow). The harvested bone graft is held in situ with a pair of tweezers. (B) After mobilization, the bone graft $(*)$ is turned around. The entire spongiosa is intensively vascularized by multiple microvesels (blue staining).

(21.5-27.8) and a mean acromioclavicular joint ramus acromialis distance of $7.4 \mathrm{~mm}(6.0-8.5)$. The mean coracoacromial ligament length (defining the transplantation radius of the vascularized bone graft) was $34.6 \mathrm{~mm}$ (28.8-36.4). The mean vascular diameter of the acromial branch at the level of the coracoid process was $2.4 \mathrm{~mm}$ (1.5-2.8). Details are listed in the Table and shown in Figure 5.

\section{Discussion}

Huntington recognized the advantages of vascularized bone grafts in $1905 .^{12}$ With preserved life and function of osteocytes and osteoblasts, graft incorporation is facilitated without the usual replacement of the graft by "creeping substitution". ${ }^{24}$ Graft resorption, a common problem seen in conventional bone transfers, can largely be prevented. ${ }^{4,6,39}$

The main objective of a vascularized bone graft is to preserve the life and function of osteoblastic cells. To achieve this goal, the ideal pedicled bone graft should have a high anatomical consistency of its nutrient vessel to allow reproducible and safe surgical graft harvesting with maintenance of its microcirculation. Moreover, graft withdrawal should lead to minimal morbidity at the donor site. ${ }^{36}$ The main indication for a pedicled bone graft is bony defects in a hypovascular zone (eg, in the context of avascular pseudarthrosis or osteonecrosis). Plenty of vascularized bone grafts are available to treat disorders around the wrist, ${ }^{25,28}$ but descriptions of pedicled bone transfers at the shoulder are mainly anecdotal. In 1987, Rindell ${ }^{26}$ used a deltoid muscle-pedicled bone graft to treat avascular necrosis of the humeral head in 1 patient. Eighteen months after surgery, improved joint function and radiological signs of graft integration in the recipient bed were observed. Recently, a Japanese research group proposed vascularized bone transfer from the scapula which they implanted successfully in a 27-year-old patient with a corticosteroidinduced osteonecrosis of the humeral head. ${ }^{14}$

The theoretical advantages of vascular bone grafts for treatment of established or impending necrosis of the humeral head are convincing. Enhancing the revascularization process by providing a new blood supply to an avascular area makes sense. Improved mechanical support of subchondral bone may help to prevent collapse of the humeral head. ${ }^{34}$

Nonetheless, due to the small number of cases available for analyses, it remains uncertain if it was the vascularized bone graft that resulted in the favorable outcomes in the case reports mentioned above. It is possible that core decompression (carried out by drilling of the humeral head for graft introduction) gave rise to the healing stimulus. ${ }^{16,20}$ From this viewpoint, the above-mentioned extended surgical techniques, which inevitably will lead to some collateral damage, seem to be too hazardous.

A recent study focused on the acromial branch as a potential bleeding source during arthroscopic surgery ${ }^{41}$; however, we are not aware of proposals to use it as a pedicle for vascularized bone grafting from the acromion. However, this graft site has the advantage of easy accessibility and limited morbidity at the donor site. Graft harvesting, if reasonably performed, should not be much more harmful than open acromioplasty. The present study revealed high reliability of the anatomical course of the acromial branch, which was consistently found to perfuse the harvested bone graft.

As described by Neer, ${ }^{22}$ the anterior acromion is best exposed through a limited anterior deltoid-splitting approach. Detachment of the deltoid should be kept to a minimum and if needed only with its tendinous origin, providing a rim of tissue for reinsertion. The coracoacromial branches (which emanate from the acromial artery and enter the acromion near the 


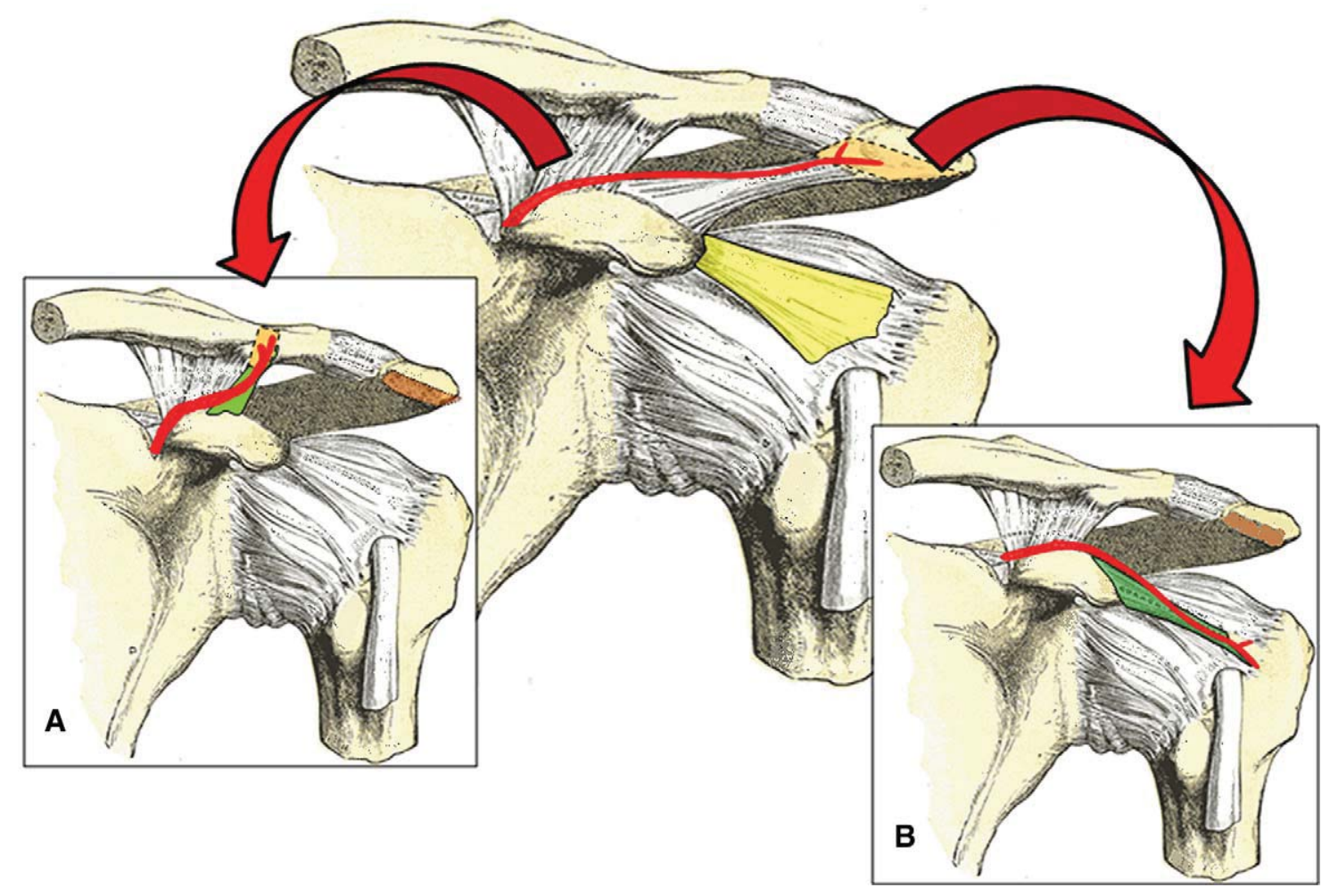

Figure 4 The potential spectrum of application for a vascularized bone graft from the acromion is shown. Depicted are a transfer to the calvicula (A) for the treatment of atrophic nonunion and transfer to the humeral head, for instance, in osteonecrosis (B). Note the coracohumeral ligament (highlighted in yellow), which can serve as guiding structure for the transfer to the humeral head.

Table Topographic relationship of the acromial branch to anatomical landmarks

\begin{tabular}{lcclll}
\hline & $N$ & Mean $(\mathrm{mm})$ & $95 \% \mathrm{CI}$ & $\mathrm{SE}$ & $\mathrm{SD}$ \\
\hline CORAD & 30 & 23.2 & $22.43-23.91$ & 0.362 & 2.018 \\
ACRAD* & 30 & 7.2 & $6.92-7.54$ & 0.152 & 0.844 \\
Diameter & 30 & 2.4 & $2.20-2.56$ & 0.089 & 0.495 \\
COALL & 30 & 33.9 & $32.70-35.04$ & 0.573 & 3.191 \\
\hline
\end{tabular}

CORAD, coracoid ramus acromialis distance; $A C R A D$, acromioclavicular joint ramus acromialis distance; COALL, coracoacromial ligament length.

* The diameter of the acromial branch at the level of the coracoids process.

insertion of the CAL) are in no danger as long as the dissection does not exceed the thickness of the deltoid insertion. As an alternative, a standard deltopectoral approach can be used. The deltoid is gently elevated with the arm held in flexion, abduction and some external rotation. One or 2 Hohmann retractors, introduced from below through the insertion of the deltoid muscle on the acromion, allow exposure of the anteroinferior edge of the acromion and harvest of the bone graft. With a thin, sharp osteotome, directed horizontally in the posterolateral direction, a wedge-shaped bone graft is harvested that comprises the anterior and lateral edge of the acromial undersurface. In order to allow accurate deltoid refixation only a limited bone wedge can be obtained. Its dimensions vary depending on the size of the patient and the

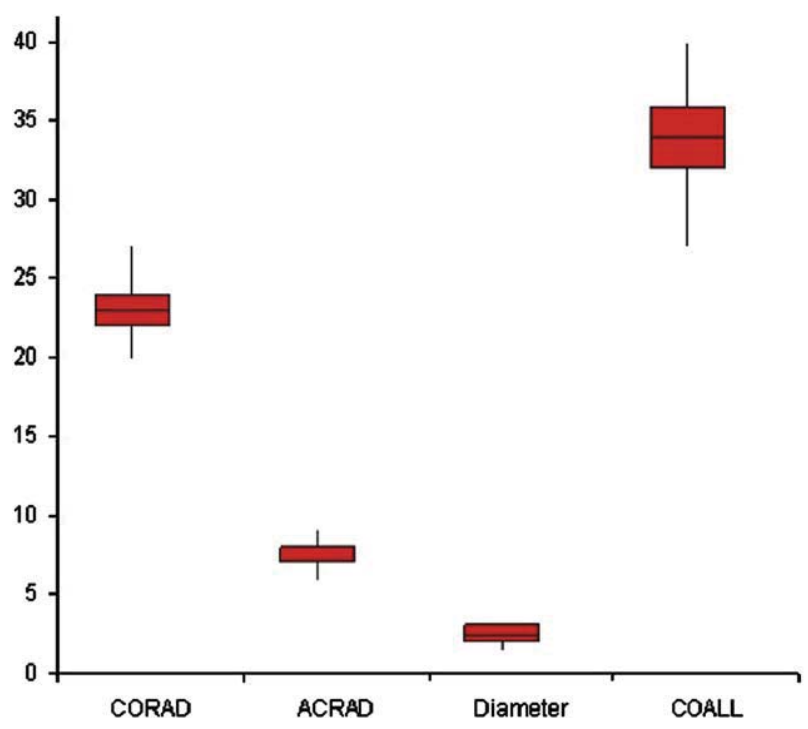

Figure 5 CORAD, coracoid ramus acromialis distance; ACRAD, acromioclavicular joint ramus acromialis distance; the diameter of the acromial branch at the level of the coracoids process; COALL, coracoacromial ligament length. All parameters are graded in millimeters.

individual anatomy of the acromion. Nevertheless, as already described by $\mathrm{Neer}^{22}$ in 1972, a bone wedge about 6-10 mm thick anteriorly and $20 \mathrm{~mm}$ long should be available in most 
patients, which corresponds to the graft sizes found in our investigation. At this location, the bone is quite hard and of good quality. Its wedge profile allows easy interposition between bone fragments (for instance the tuberosities) and simplifies introduction into the humeral head.

The main weak point of this graft site is the limited bone volume available for harvesting. Major bone losses cannot be reconstructed by this source. Furthermore, the coracoacromial arterioles were sometimes quite fragile. Dissection of this region was found to be the critical step during graft withdrawal. To protect these small vessels, insertion of the CAL must be preserved. By doing so, the bone graft can be harvested under meticulous maintenance of its nutrient branches. Moreover, the CAL protects the pedicle from traction forces during mobilization and transposition of the graft. With the CAL still attached to the coracoids, one could worry that it would form a tether of the shoulder joint and restrict range of motion. However, there is already a pre-existing physiological connection between the humeral head and the coracoid in form of the coracohumeral ligament (Fig. 4, highlighted in yellow). With the bone graft introduced at the humeral insertion of the coracohumeral ligament, restriction of shoulder mobility is unlikely. We performed this procedure in 5 specimens, and the range of shoulder motion stayed unlimited. After graft transposition, range of shoulder motion has to be intraoperatively assessed. If the CAL gets stretched (especially in adduction and external rotation) or a greater transposition range is needed, lengthening of the CAL with a $\mathrm{Z}$ plasty can be performed, or it can be completely released at the base of the coracoid process.

Previous shoulder surgery (particularly a history of an open or arthroscopic acromioplasty) is a major contraindication for use of this graft, because it inevitably leads to destruction of the coracoacromial branches.

Complex fractures of the proximal humerus remain a challenging problem, and evidence concerning the optimal therapeutic approach is lacking. Malunion, nonunion, stiffness, and avascular necrosis are potential complications. The risk of post-traumatic avascular necrosis is determined primarily by fracture type and is aggravated by inappropriate dissection of soft tissue during open reduction and internal fixation. The prevalence is $0-33 \%$ after open reduction and internal fixation of 3-part fractures and up to $80-90 \%$ for 4-part fractures. ${ }^{10,11,15,30}$ In a retrospective evaluation of 25 patients with a partial or complete collapse of the humeral head due to post-traumatic avascular necrosis, Gerber et al found a relevant disability of the involved shoulder with ageand sex-matched shoulder function of only $51 \% .^{7}$

Several authors reported favorable outcomes predominantly in terms of pain relief in the treatment of such fractures with hemiarthroplasty ${ }^{2,19}$; however, young patients with complex fractures carrying the risk of avascular necrosis remain a major problem. It is in this population (in which prosthetic replacement should be avoided whenever possible) in which such a bone graft could be indicated. If intraoperative drilling of the head fragment does not reveal bleeding, but correct osteosynthesis seems feasible, an additional blood supply provided by the vascularized bone graft may help to prevent future head collapse. If primary prosthetic replacement is unavoidable, interposition of the vascularized bone graft between the greater and lesser tuberosity may help to impede postoperative migration, nonunion, or resorption of the tuberosities. Aside from problems of fixation, we are convinced that these complications are also partially caused by an impaired vascularity. Another potential application is revision of avascular nonunion of a clavicular fracture.

This study has several limitations. The setting of a purely anatomical investigation does not allow us to draw any conclusions regarding its clinical usefulness. According to Bastian and Hertel, ${ }^{3}$ not all initially ischemic humeral heads develop avascular necrosis and collapse. Otherwise some of the initially perfused heads necrotize secondary. Thus precise criteria for decision-making if vascularized bone grafting should be performed or not are lacking. Furthermore, with apparent osteonecrosis, we cannot predict when in its evolution such an intervention should be considered and how the treating surgeon should position the graft within the necrotic segment.

Despite their theoretical advantages, the indications and results of vascularized bone transfers are currently a matter of debate. While some authors reported promising results of free vascularized fibular grafting in the treatment of osteonecrosis of the femoral head, ${ }^{34,35}$ others raised concern that revascularization may promote femoral head collapse. ${ }^{8}$ Revascularization initiates a competition between restoration of bony stability by creeping substitution and impending head collapse by resorption of necrotic bone trabeculae. As the mechanical load of the shoulder is less than that of the weight-bearing lower extremity, this "race" may be more advantageous for the restoration process. However, as already mentioned, this is an anatomical study without clinical results and cannot provide any further evidence to support this assumption.

Additional investigations are mandatory to clarify all these open questions.

\section{Conclusion}

We demonstrated the feasibility of vascularized bone grafting from the acromion pedicled on the acromial branch of the thoracoacromial artery. The acromial branch revealed a consistent course over the coracoid process with a close topographic relationship to anatomical landmarks. Near the acromial insertion of the CAL, the acromial branch gives origin to coracoacromial arterioles, which reliably supply the anterior part of the acromion. To preserve these vessels, the CAL has to stay in continuity with the acromion. The 
CAL, which protects the pedicle from traction forces, mainly limits the range of graft transposition; however, the distance is more than sufficient to allow tension-free transfer of the vascularized bone graft to the humeral head, as well as to the lateral two-thirds of the clavicle. Our data support that a vascular bone graft from the acromion, pedicled on the acromial branch, is technically feasible. This technique may have the potential to be a new joint-preserving procedure for osteonecrosis of the humeral head or may be helpful in the revision of a clavicular pseudarthrosis. However, its clinical usefulness needs to be further investigated.

\section{Disclaimer}

No author, their immediate family, and any research foundation with which they are affiliated have received any financial payments or other benefits from any commercial entity related to the subject of this article.

\section{References}

1. Aldridge JM III, Urbaniak JR. Avascular necrosis of the femoral head: role of vascularized bone grafts. Orthop Clin North Am 2007;38: 13-22. doi:10.1016/j.ocl.2006.10.012

2. Bastian JD, Hertel R. Osteosynthesis and hemiarthroplasty of fractures of the proximal humerus: outcomes in a consecutive case series. J Shoulder Elbow Surg 2009;18:216-9. doi:10.1016/j.jse.2008. 09.015

3. Bastian JD, Hertel R. Initial post-fracture humeral head ischemia does not predict development of necrosis. J Shoulder Elbow Surg 2008;17: 2-8. doi:10.1016/j.jse.2007.03.026

4. Bauer TW, Muschler GF. Bone graft materials. An overview of the basic science. Clin Orthop Relat Res 2000:10-27.

5. Dittmann V, Fasel JH, Hornung JP. Verwendung von Leichen und Leichenteilen in der medizinischen Forschung sowie Aus-, Weiterund Fortbildung. Senat der Schweizerischen Akademie der Medizinischen Wissenschaften (SAMW) 2008. (http://www.samw.ch/en/ News/News.html)

6. Doi K, Tominaga S, Shibata T. Bone grafts with microvascular anastomoses of vascular pedicles: an experimental study in dogs. J Bone Joint Surg Am 1977;59:809-15.

7. Gerber $\mathrm{C}$, Hersche $\mathrm{O}$, Berberat $\mathrm{C}$. The clinical relevance of posttraumatic avascular necrosis of the humeral head. J Shoulder Elbow Surg 1998;7:586-90.

8. Glimcher MJ, Kenzora JE. The biology of osteonecrosis of the human femoral head and its clinical implications. III. Discussion of the etiology and genesis of the pathological sequelae; commments on treatment. Clin Orthop Relat Res 1979:273-312.

9. Grabherr S, Djonov V, Friess A, Thali MJ, Ranner G, Vock P, et al. Postmortem angiography after vascular perfusion with diesel oil and a lipophilic contrast agent. Am J Roentgenol 2006;187:W515-23. doi: 10.2214/AJR.05.1394

10. Hawkins RJ, Angelo RL. Displaced proximal humeral fractures. Selecting treatment, avoiding pitfalls. Orthop Clin North Am 1987;18: 421-31.
11. Hawkins RJ, Kiefer GN. Internal fixation techniques for proximal humeral fractures. Clin Orthop Relat Res 1987:77-85.

12. Huntington TW. VI. Case of bone transference: Use of a segment of fibula to supply a defect in the tibia. Ann Surg 1905;41:249-51.

13. Johnson EW Jr, Collins HR. Nonunion of the clavicle. Arch Surg 1963;87:963-6.

14. Kawamura K, Kawate K, Yajima H, Kobata Y, Takakura Y. Vascularized scapular grafting for treatment of osteonecrosis of the humeral head. $\mathrm{J}$ Reconstr Microsurg 2008;24:559-64. doi:10.1055/s-0028-1090621

15. Ko JY, Yamamoto R. Surgical treatment of complex fracture of the proximal humerus. Clin Orthop Relat Res 1996:225-37.

16. LaPorte DM, Mont MA, Mohan V, Pierre-Jacques H, Jones LC, Hungerford DS. Osteonecrosis of the humeral head treated by core decompression. Clin Orthop Relat Res 1998:254-60.

17. Lee CK, Hansen HR. Post-traumatic avascular necrosis of the humeral head in displaced proximal humeral fractures. J Trauma 1981;21:788-91.

18. Minami A, Ogino T, Sakuma T, Usui M. Free vascularized fibular grafts in the treatment of congenital pseudarthrosis of the tibia. Microsurgery 1987;8:111-6.

19. Moeckel BH, Dines DM, Warren RF, Altchek DW. Modular hemiarthroplasty for fractures of the proximal part of the humerus. J Bone Joint Surg Am 1992;74:884-9.

20. Mont MA, Maar DC, Urquhart MW, Lennox D, Hungerford DS. Avascular necrosis of the humeral head treated by core decompression. A retrospective review. J Bone Joint Surg Br 1993;75:785-8.

21. Moore JR, Weiland AJ, Daniel RK. Use of free vascularized bone grafts in the treatment of bone tumors. Clin Orthop Relat Res 1983: 37-44.

22. Neer CS II. Anterior acromioplasty for the chronic impingement syndrome in the shoulder: a preliminary report. J Bone Joint Surg Am 1972;54:41-50.

23. Neer CS II. Nonunion of the clavicle. J Am Med Assoc 1960;172: 1006-11.

24. Phemister DB. The fate of transplanted bone and regenerative power of its various constituents. Surg, Gynec Obstet 1914;19: 3003-333.

25. Pistre V, Reau AF, Pelissier P, Martin D, Baudet J. [Vascularized bone pedicle grafts of the hand and wrist: literature review and new donor sites]. Chir Main 2001;20:263-71.

26. Rindell K. Muscle pedicled bone graft in revascularization of aseptic necrosis of the humeral head. Ann Chir Gynaecol 1987;76:283-5.

27. Rockwood CA, Lyons FR. Shoulder impingement syndrome: diagnosis, radiographic evaluation, and treatment with a modified Neer acromioplasty. J Bone Joint Surg Am 1993;75:409-24.

28. Roux JL. [Vascularized bone transfers in the wrist and hand]. Chir Main 2003;22:173-85.

29. Rowe CR. An atlas of anatomy and treatment of midclavicular fractures. Clin Orthop Relat Res 1968;58:29-42.

30. Savoie FH, Geissler WB, Vander Griend RA. Open reduction and internal fixation of three-part fractures of the proximal humerus. Orthopedics 1989;12:65-70.

31. Scully SP, Aaron RK, Urbaniak JR. Survival analysis of hips treated with core decompression or vascularized fibular grafting because of avascular necrosis. J Bone Joint Surg Am 1998;80:1270-5.

32. Taylor GI, Miller GD, Ham FJ. The free vascularized bone graft. A clinical extension of microvascular techniques. Plast Reconstr Surg 1975;55:533-44.

33. Thiel W. Die Konservierung ganzer Leichen in natürlichen Farben. Ann. Anat 1992:185-95.

34. Urbaniak JR, Coogan PG, Gunneson EB, Nunley JA. Treatment of osteonecrosis of the femoral head with free vascularized fibular grafting. A long-term follow-up study of one hundred and three hips. J Bone Joint Surg Am 1995;77:681-94.

35. Urbaniak JR, Harvey EJ. Revascularization of the femoral head in osteonecrosis. J Am Acad Orthop Surg 1998;6:44-54

36. Vail TP, Urbaniak JR. Donor-site morbidity with use of vascularized autogenous fibular grafts. J Bone Joint Surg Am 1996;78:204-11. 
37. Weiland AJ. Vascularized bone transfers. Instr Course Lect 1984;33: 446-60

38. Weiland AJ. Current concepts review: vascularized free bone transplants. J Bone Joint Surg Am 1981;63:166-9.

39. Weiland AJ, Moore JR, Daniel RK. Vascularized bone autografts. Experience with 41 cases. Clin Orthop Relat Res 1983:87-95.
40. Weiland AJ, Weiss AP, Moore JR, Tolo VT. Vascularized fibular grafts in the treatment of congenital pseudarthrosis of the tibia. J Bone Joint Surg Am 1990;72:654-62.

41. Yepes H, Al-Hibshi A, Tang M, Morris SF, Stanish WD. Vascular anatomy of the subacromial space: a map of bleeding points for the arthroscopic surgeon. Arthroscopy 2007;23:978-84. doi:10.1016/j.arthro.2007.03.093 